\title{
微生物粘附加速钻长石溶解的直接证据: 透析法实验研究
}

周跃飞 ${ }^{(1)}$, 王汝成 ${ }^{(1 *}$, 陆现彩 $^{(1)}$

(1) 内生金属成矿机制研究国家重点实验室, 南京大学地球科学与工程学院, 南京 210093;

(2) 合肥工业大学资源与环境工程学院, 合肥 230009

* 联系人, E-mail: rcwang@nju.edu.cn

收稿日期: 2009-12-14; 接受日期: 2010-07-06

国家重点基础研究发展计划(编号：2007CB815603)、国家自然科学基金(批准号：40802015)和教育部博士点基金(批准号：20050284043, 20050284044)资助

\begin{tabular}{|c|c|}
\hline 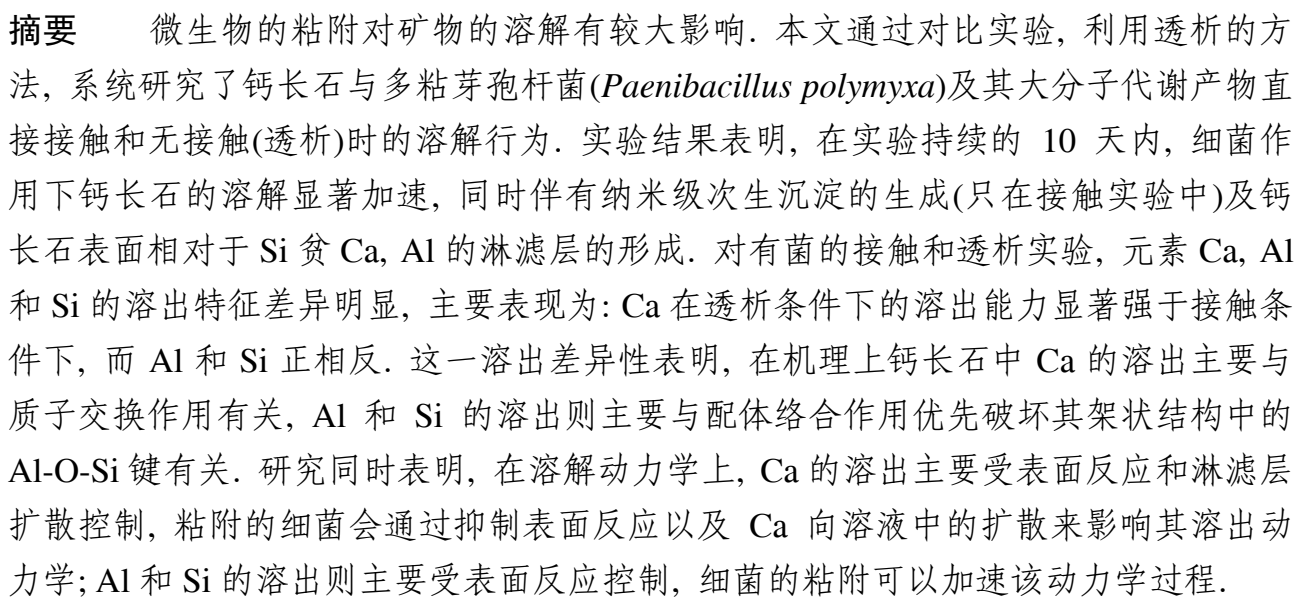 & $\begin{array}{l}\text { 关键词 } \\
\text { 钻长石 } \\
\text { Paenibacillus polymyxa } \\
\text { 粘附 } \\
\text { 溶解 } \\
\text { 透析法 }\end{array}$ \\
\hline
\end{tabular}

自然界的土壤和水体中广泛分布着微生物，微 生物通常会通过范德华力、静电相互作用力、氢键等 粘附于矿物、岩石等固体物质表面 ${ }^{[1,2]}$. 这种粘附作用 往往能够直接影响矿物或岩石表面的理化性质, 从 而改变其溶解行为 ${ }^{[3 \sim 9]}$. 如粘附于矿物或岩石表面的 微生物的代谢物在扩散速率较低时, 会在其周围积 聚, 形成“液膜”,[5 7,9]; 同时, 大量微生物及代谢物也 可以通过化学键的作用相互交联, 在矿物或岩石表 面形成生物膜 ${ }^{[10]}$. 无论是液膜还是生物膜, 往往均具
有特殊的物质组成及含量(如氢离子、有机配体等), 因此会对矿物或岩石的溶解能力产生较大影响. 以 自然界广泛存在的长石为例, 在微生物存在时, 其表 面很容易形成液膜或生物膜 ${ }^{[5,8 \sim 10]}$, 由于质子交换(即 酸解作用)和配体络合是微生物及其代谢物促进长石 溶解的两种主要机制 ${ }^{[5,11 ~ 14]}$, 因此, 微生物在长石表 面的粘附对长石溶解具有重要影响 ${ }^{[8 \sim 10]}$.

然而, 目前关于微生物粘附影响长石溶解的研 究大多还停留在定性描述阶段. 这是因为在长石-溶

英文引用格式: Zhou Y F, Wang R C, Lu X C. Anorthite dissolution promoted by bacterial adhesion: Direct evidence from dialytic experiment. Sci China Earth Sci, 2011, 54: 204-211, doi: 10.1007/s11430-010-4068-y 
液-微生物三相系中, 微生物通常通过三种方式影响 长石溶解: (1) 与长石接触时的粘附效应; (2) 与代谢 产物一同进入溶液, 通过改变溶液理化性质 $(\mathrm{pH}$ 、有机 配体种类及浓度等) 影响长石溶解的溶液效应 ${ }^{[5,9,10]}$; (3) 可能存在的粘附效应和溶液效应的协同效应. 而 在通常的室内模拟实验中, 长石和微生物在溶液相 中是直接接触的 ${ }^{[8 \sim 10]}$, 这样的实验设计难以区分溶液 效应和粘附效应(以及可能的协同效应), 其结果是使 得在探讨粘附对长石中不同元素溶出的作用机制(质 子交换和配体络合)时, 难以消除溶液效应的影响, 进而通过定量的方式进行解释. 此外, 在溶解动力学 上, 由于液膜或生物膜的存在, 长石中各元素的溶出 动力学模式可能也会发生改变, 已有的动力学模式 如表面反应控制、扩散控制、淋滤层-表面反应控制 等 ${ }^{[15 ~ 20]}$, 是否仍适用于粘附条件下的溶解, 也还有 待研究.

据此, 为有效区分微生物影响长石溶解的溶液 效应和粘附效应, 本文在前人实验研究的基础上 ${ }^{[10]}$, 改进实验设计, 引入透析方法 ${ }^{[21]}$, 通过对比多粘芽孢 杆菌(Paenibacillus polymyxa)直接接触和无接触条件 下长石溶解行为的差异, 研究微生物及其代谢产物 的粘附行为在长石溶解过程中的作用, 并探讨粘附 作用的动力学意义.

\section{1 实验材料及方法}

\section{1 实验材料}

实验用钙长石挑选自福建莆田岱前山层状角闪 辉长岩. 供试长石的粒径约 80 200 目 (d=0.075 0.180 $\mathrm{mm}$ ), 其中 $\mathrm{An}$ 的质量百分含量约 $90 \%{ }^{[22]}$. 实验前先 用酒精超声清洗 5 次(每次 $10 \mathrm{~min}$ ), 然后在 $\mathrm{pH}=4.0$ 的稀盐酸溶液中浸泡 $2 \mathrm{~h}$, 再用去离子水超声清洗至 溶液澄清. 最后将钙长石在 $70^{\circ} \mathrm{C}$ 下烘干后使用.

实验用细菌为多粘芽孢杆菌 (Paenibacillus polymyxa), 选取该细菌作为实验菌的依据是该菌株 为表生条件下广泛存在的化能异养型兼性厌氧菌, 与一般的化能异养菌相似, 其在生长过程中能产生 大量的有机酸(如甲酸、乙酸、乳酸、柠檬酸)及多聚 糖等, 这些产物和细菌自身均有可能促进矿物溶解, 如已有研究表明该细菌及其代谢产物对铝土矿中杂质 $\mathrm{Ca}$ 的溶出具有显著促进作用 ${ }^{[23]}$. 因此, 选用该细菌, 可以更真实地反应自然界的地质微生物过程 ${ }^{[23 \sim 25]}$. 细
菌购自中国普通微生物菌种保藏管理中心(CGMCC). 菌种经活化后用牛肉蛋白培养基(牛肉亳 $0.3 \%$, 蛋白 胨 1\%, $\mathrm{NaCl} 0.5 \%$ )(W/V)(根据 CGMCC 的建议培养基 配制)进行连续培养后保存待用.

为了有效阻止细菌及其高分子量代谢物(如多聚 糖)粘附在长石表面, 本实验使用透析袋包裹钙长石 颗粒. 透析袋的材质为纤维素聚酯, 孔径为 14000 (即 只能透过分子量低于 14000 的物质, 物理孔径约 25 A). 多粘芽狍杆菌代谢产生的高分子量代谢物主要 为多聚糖, 多为分子量在 17000 左右的中糖(约占多 聚糖质量的 $80 \%$ ) 和糖醛酸(约 $16.5 \%)^{[25]}$, 因此细菌及 其多聚糖类代谢物均不能通过透析袋与矿物直接接 触. 实验前, 首先将透析袋在 $2 \%(\mathrm{~W} / \mathrm{V})$ 的 $\mathrm{NaHCO}_{3}$ 和 $1 \mathrm{mmol} \mathrm{L}^{-1}$ 的 $\operatorname{EDTA}(\mathrm{pH}=8.0)$ 中煮沸 $10 \mathrm{~min}$, 用去离 子水清洗后, 再于 $1 \mathrm{mmol} \mathrm{L}^{-1}$ 的 $\mathrm{EDTA}(\mathrm{pH}=8.0)$ 中煮 沸 $10 \mathrm{~min}$, 用二次去离子水清洗后备用.

\section{2 实验方法}

本研究设计了三组实验: (1) 无培养基、无透析 袋的无菌实验(编号 a1); (2) 有培养基、有细菌, 不使 用透析袋实验(编号 a2); (3) 有培养基、有细菌, 使用 透析袋实验(编号 a3). 每组实验做三个重复.

具体实验步骤为: 首先称取钻长石 9 份, 每份 $0.80 \mathrm{~g}$, 其中 3 份装入透析袋并用橡皮筋封口; 将 9 份 钻长石样品连同配制好的改性布氏培养基(蔗糖 $0.5 \%$, 酵母膏 $0.02 \%, \mathrm{KH}_{2} \mathrm{PO}_{4} 0.05 \% ，\left(\mathrm{NH}_{4}\right)_{2} \mathrm{SO}_{4} \quad 0.1 \%$, $\left.\mathrm{MgSO}_{4} \cdot 7 \mathrm{H}_{2} \mathrm{O} \quad 0.015 \%\right)(\mathrm{W} / \mathrm{V})^{[26]}$ 在 $121^{\circ} \mathrm{C}$ 的条件下蒸 汽灭菌 $20 \mathrm{~min}$; 将灭菌后的培养基分装入已消毒的 聚乙烯三角瓶中, 每瓶 $100 \mathrm{~mL}$, 其中三只直接加入 经灭菌的钙长石粉末(a2), 另三只加已装入透析袋的 灭菌长石(a3); 量取 $2 \mathrm{~mL}$ 经牛肉蛋白培养基培养的 P. polymyx 菌液, 以 $5000 \mathrm{rpm}$ 的速率离心分离 $10 \mathrm{~min}$ 后，使用无菌二次水清洗分离出的沉淀物 5 遍，随后 将之接种到实验的三角瓶中; 此外, 尚需使用灭菌二 次水加等量钙长石的方法做无菌对比实验 (a1). 将所 有三组 9 个三角瓶置于恒温摇床上, 在 $30^{\circ} \mathrm{C}, 100 \mathrm{rpm}$ 的条件下进行 $240 \mathrm{~h}$ 的连续溶解实验.

\section{3 取样及测试方法}

在实验过程中, 分别在第 $0,18,48,96,168$ 和 $240 \mathrm{~h}$ 时取样, 每次取上清液 $5 \mathrm{~mL}$, 测定 $\mathrm{pH}$ 值. 为了 脱附细菌及其代谢产物表面吸附的 $\mathrm{Ca}, \mathrm{Al}$ 及 $\mathrm{Si}$, 溶液 
随后用 $1 \mathrm{~mol} \mathrm{~L}^{-1} \mathrm{HNO}_{3}$ 酸化至 $1.00<\mathrm{pH}<2.00^{[27]}$, 酸 化溶液放置 $1 \mathrm{~h}$ 后(中间间歇摇振以使脱附充分), 在 高速离心机上以 $10000 \mathrm{rpm}$ 的速度离心 $10 \mathrm{~min}$, 抽取 上清液采用电感耦合等离子发射光谱(ICP-AES)法测 定 $\mathrm{Ca}, \mathrm{Al}, \mathrm{Si}$ 浓度. 仪器型号为 JY38S, 测试精度为: Ca $0.75 \mathrm{nmol} \mathrm{L}^{-1}$, Al $0.15 \mu \mathrm{mol} \mathrm{L}^{-1}$, Si $0.11 \mu \mathrm{mol} \mathrm{L}^{-1}$.

为了考察长石溶解前后表面化学结构的变化, 将残余钻长石烘干后进行微区激光拉曼光谱测试, 仪器型号为 Renishaw RM2000, 采用 $\mathrm{Ar}$ 离子激光器 为激发光源, 激光波长为 $514 \mathrm{~nm}$, 狭缝宽 $40 \mu \mathrm{m}$, 扫 描波数范围为 $100 \sim 1200 \mathrm{~cm}^{-1}$, 扫描时间为 $10 \mathrm{~s}$. 为了 观察实验前后长石的表面形貌变化, 将反应前后的 长石冷冻干燥后进行扫描电镜观察. 采用的仪器为 JSM-6300 型扫描电子显微镜, 测试条件为: 加速电 压 $20 \mathrm{kV}$, 速流 $80 \mu \mathrm{A}$.

ICP-AES 和激光拉曼测试在南京大学内生金属 矿床成矿机制研究国家重点实验室进行, 扫描电镜 观察在中国科学院南京地质古生物研究所现代古生 物学和地层学国家重点实验室进行.

\section{2 实验结果}

\section{1 细菌生长与溶液 $\mathrm{pH}$ 值}

根据周跃飞等 ${ }^{[28]}$ 在同样培养条件下测定的 $P$. polymyxa 生长曲线, 在培养约 $18 \mathrm{~h}$ 后, 细菌进入生长 稳定期, 此时的最大细菌量约为(1 2) $\times 10^{7}$ 个 $\mathrm{mL}^{-1}$, $48 \mathrm{~h}$ 后进入衰亡期.

无菌实验 $(\mathrm{a} 1)$ 的 $\mathrm{pH}$ 值在整个实验过程中变化不 大(图 1). 有菌实验中, 溶液 $\mathrm{pH}$ 值随细菌生长出现有 规律的变化: 在 $0 \sim 18 \mathrm{~h}$, 从 7.0 迅速下降至 $4.3 ; 18 \mathrm{~h}$ 后, 下降速率变缓并逐渐趋于稳定; $96 \mathrm{~h}$ 后开始缓慢 升高, 其中实验 $\mathrm{a} 2$ 的升高幅度(到结束时升高了约 0.3 )比 $\mathrm{a} 3$ 小(到实验结束时升高了约 1.0). $\mathrm{pH}$ 值在实 验后期逐渐升高的现象, 与 Welch 等 ${ }^{[29]}$ 的发现相似.

\section{2 元素浓度}

图 2 表示了实验溶液中䥻长石的三种主要元素 $(\mathrm{Ca}, \mathrm{Al}, \mathrm{Si})$ 浓度的变化情况. 可以看出, 总体上, 实 验结束时三种元素的浓度均在有菌条件下增加更多. 从浓度变化过程上看, 无菌条件下各元素浓度变化 不具有阶段性, 而有菌条件下各元素浓度变化阶段 性明显, 具体体现为:

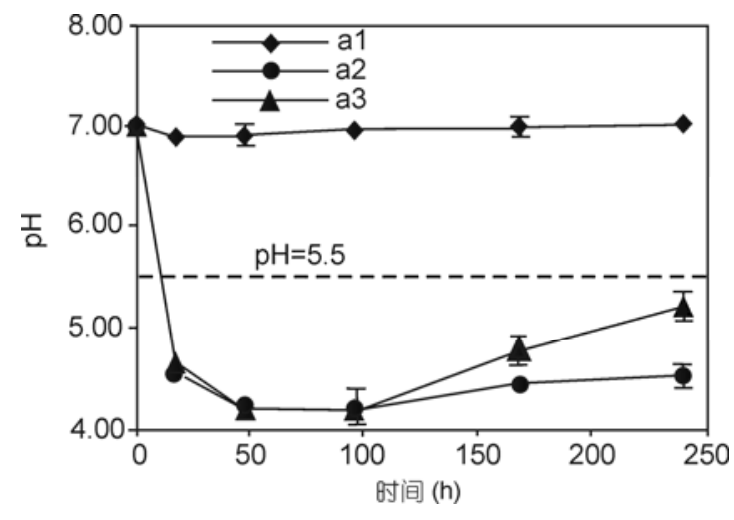

图 1 实验溶液 $\mathrm{pH}$ 随时间的变化

（1）无菌实验. 实验初始时候 $\mathrm{Ca}$ 浓度增幅较大, 随后则逐渐降低, 到实验后期浓度几乎不再变化(图 2(a)), 浓度随时间的变化具有对数特征(表 1). Al 和 $\mathrm{Si}$ 的浓度则保持较低的恒定增幅(图 2(b)), 浓度-时间 总体上呈线性关系(表 1). 实验结束时 $\mathrm{Ca}$ 浓度增加最 多, $\mathrm{Al}$ 和 $\mathrm{Si}$ 均增加较少.

(2) 有菌实验. 三种元素浓度变化均可显著分为 两个阶段, 即初始的 $18 \mathrm{~h}$ (与细菌生长的迟缓期和对 数生长期相对应) 和之后的时间段 (与细菌生长的稳 定期和衰亡期对应). 在初始 $18 \mathrm{~h}$, 三种元素浓度变 化均不大; $18 \mathrm{~h}$ 后, 各元素浓度均明显上升. 这种浓 度变化的阶段性与 Hutchens 等 ${ }^{[30]}$ 的实验结果相似. 在第二阶段, 三种元素浓度变化的特征与无菌条件 下相似, 即 $\mathrm{Ca}$ 的浓度上升速率为开始较大, 随后逐 渐降低, 浓度-时间具有对数关系, $\mathrm{Si}$ 和 $\mathrm{Al}$ 保持稳定 上升, 浓度-时间具有线性关系(表 1). 实验结束后 $\mathrm{Ca}$ 浓度上升最多, $\mathrm{Si}$ 次之, $\mathrm{Al}$ 最少. 接触和透析实验中 三种元素浓度变化规律均相似, 两组实验的差异性 主要体现为实验结束时, $\mathrm{Ca}$ 在透析实验中浓度增加 更多, 而 $\mathrm{Al}$ 和 $\mathrm{Si}$ 在接触实验中浓度增加更多.

\section{3 表面形貌}

利用扫描电子显微镜对钙长石样品在实验前后 的微区形貌进行了观察. 从图 3(a)及 3(b) 可以看出, 溶解前的钻长石表面棱角比较分明, 粗䊁度较高. 无 菌条件下溶解 10 天后, 钙长石表面变得较为平整, 其表面的微细颗粒状突起也变得圆滑(图 3(c), (d)), 但与实验前的长石相比, 二者微形貌差异不甚显著. 对有菌实验, 则无论是在接触还是透析模式下, 钙长 石的表面形貌均发生了比较明显的变化(图 3(e), (g)). 

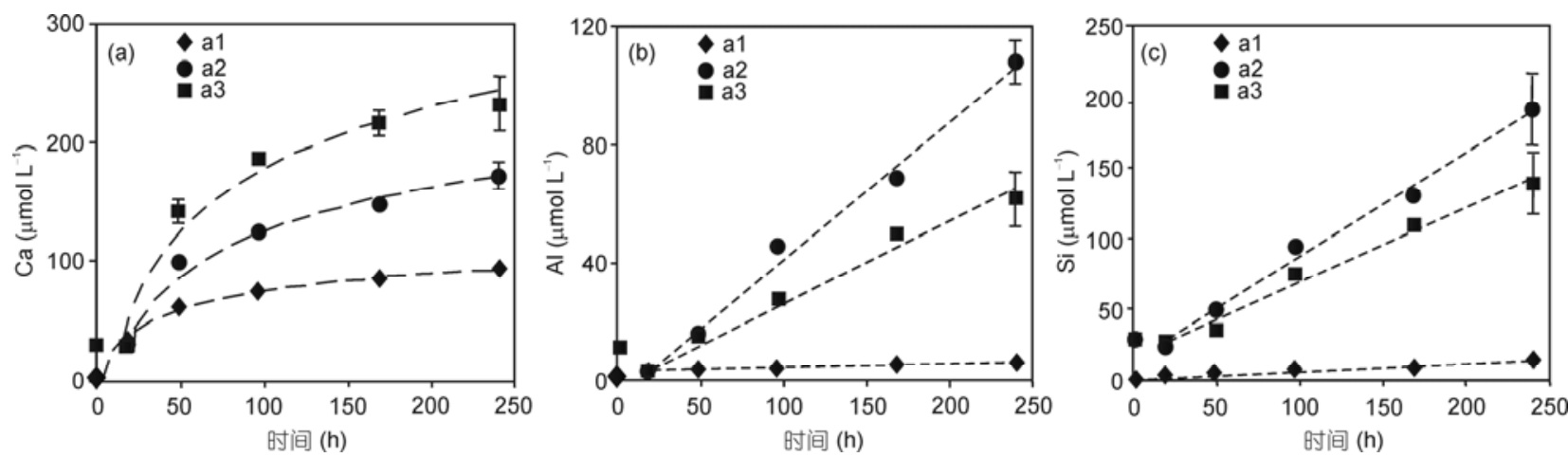

图 2 元素浓度随时间的变化

虚线为元素浓度-时间拟合曲线, 实验 $\mathrm{a} 1$ 为 $0 \sim 240 \mathrm{~h}$ 的拟合, $\mathrm{a} 2$ 和 $\mathrm{a} 3$ 为 $18 \sim 240 \mathrm{~h}$ 的拟合, 拟合方程见表 1

表 1 溶液中各溶出元素浓度 $(C)$-时间 $(t)$ 拟合方程及相关系 数 $\left(R^{2}\right)^{\mathrm{a})}$

\begin{tabular}{clcc}
\hline 元素 & \multicolumn{1}{c}{ 拟合方程 } & $R^{2}$ & $\begin{array}{c}\text { 浓度变化速率方程 } \\
(r=\mathrm{d} C / \mathrm{d} t)\end{array}$ \\
\hline \multirow{2}{*}{$\mathrm{Ca}$} & $C_{\mathrm{a} 1}=21.8 \ln t-25$ & 0.99 & $\begin{array}{c}21.8 / t \\
54 / t\end{array}$ \\
& $C_{\mathrm{a} 2}=54 \ln t-123$ & 0.98 & $75.7 / t$ \\
\hline \multirow{3}{*}{$\mathrm{A} 1$} & $C_{\mathrm{a} 3}=75.7 \ln t-169$ & 0.97 & $1.27 / t$ \\
& $C_{\mathrm{a} 1}=1.27 \ln t-0.89$ & 0.86 & 0.47 \\
& $C_{\mathrm{a} 2}=0.47 t-5.4$ & 0.99 & 0.28 \\
\hline \multirow{2}{*}{$\mathrm{Si}$} & $C_{\mathrm{a} 3}=0.28 t-2.1$ & 0.97 & 0.05 \\
& $C_{\mathrm{a} 1}=0.05 t+1.68$ & 0.91 & 0.73 \\
& $C_{\mathrm{a} 2}=0.73 t+15$ & 0.99 & 0.52 \\
\hline
\end{tabular}

a) $C$ 的单位为 $\mu \mathrm{mol} \mathrm{L}^{-1}, t$ 的单位为 $\mathrm{h}$

图 3(e)为接触实验后的钙长石表面微形貌, 可以显见 棱角处(图中 A 所示的位置)变得比较圆滑; 同时, 接 触实验中的钙长石表面还出现沉淀现象(图 3(e), (f)), 在钻长石表面较光滑的断面平台上, 大量粒径约 100 200 $\mathrm{nm}$ 的微球粒沉淀于矿物表面. 图 3(g)是透 析实验后的钙长石表面微形貌, 在机械破碎较强烈 的地方 (图中 B 所示的位置), 长石因溶解而残余了许 多粒径在 100 200 nm 的微细颗粒(图 3(h)). 图 3(f)和 (h)的微细颗粒在粒径和形貌上具有相似性, 但二者在 聚集特征上有较大差异, 前者呈葡萄状无序堆积, 后 者为串珠状残余体，这表明二者在成因上是不同的.

\section{4 激光拉曼光谱}

使用激光拉曼光谱对实验前后长石表面结构进 行了微区分析, 结果如图 4 所示. 图中 $503 \mathrm{~cm}^{-1}$ 处最 强峰是钙长石的特征峰 ${ }^{[31]}$. 在中低波数区域(100 $900 \mathrm{~cm}^{-1}$ ), 各样品的拉曼谱峰其强度和位置基本相 同. 在 $900 \sim 1200 \mathrm{~cm}^{-1}$ 高波数段主要出现三个峰: 905 ,
975 和 $1070 \mathrm{~cm}^{-1}$. 对细菌作用后的样品, 这几个峰出 现了相对于原样和无菌样的一些变化. 强度上, 峰强 普遍减弱, 其中尤以接触实验的 $905 \mathrm{~cm}^{-1}$ 峰和透析实 验的 $1070 \mathrm{~cm}^{-1}$ 峰为甚; 峰位上, 细菌作用后样品的 $975 \mathrm{~cm}^{-1}$ 峰向高频方向偏移了约 3 个波数, 而无菌溶 解实验后的样品只出现相同方向 1 个波数的偏移.

\section{3 讨论}

\section{1 细菌对长石溶解的影响}

由实验结果可知, 在无菌实验中, 经过 10 天的 溶解, 不仅溶液中 $\mathrm{Ca}$ 浓度出现了一定程度的增加, 同时矿物表面变得比较光滑, 相关的拉曼谱峰也产 生了一定的位移, 这些现象均表明钲长石在中性水 溶液中也具有一定的溶解能力. 但是, 值得注意的是, 由于曾将样品进行过酸处理，该过程可能曾导致许 多 $\mathrm{H}^{+}$吸附到矿物表面, 在清洗时由于没能充分脱附, 因此这部分残留的 $\mathrm{H}^{+}$会在随后的实验中加速长石的 溶解. 因此, 尽管本研究设定的初始溶液 $\mathrm{pH}$ 为 7.0, 但矿物表面 $\mathrm{H}^{+}$浓度是高于溶液中浓度的, “吸附性 $\mathrm{H}^{+}$”的存在及其对矿物的溶解导致出现 “质子交换作 用导致了 $\mathrm{Ca}^{2+}$ 的溶出, 但溶液 $\mathrm{pH}$ 却保持中性” 的矛 盾现象.

然而, 与无菌实验相比, 有菌实验中不仅溶液中 各元素浓度上升更多，而且矿物表面变得更加平整， 此外, 还形成了次生沉淀(接触实验)并且长石表面结 构也发生了更大的改变(拉曼光谱结果), 这些现象均 反映了细菌及其代谢产物能显著加速长石溶解. 在 接触实验中, 溶解产物除以可溶态存在外, 还以次生 沉淀物的形式附着于矿物表面(图 3(e), (f)), 在透析 

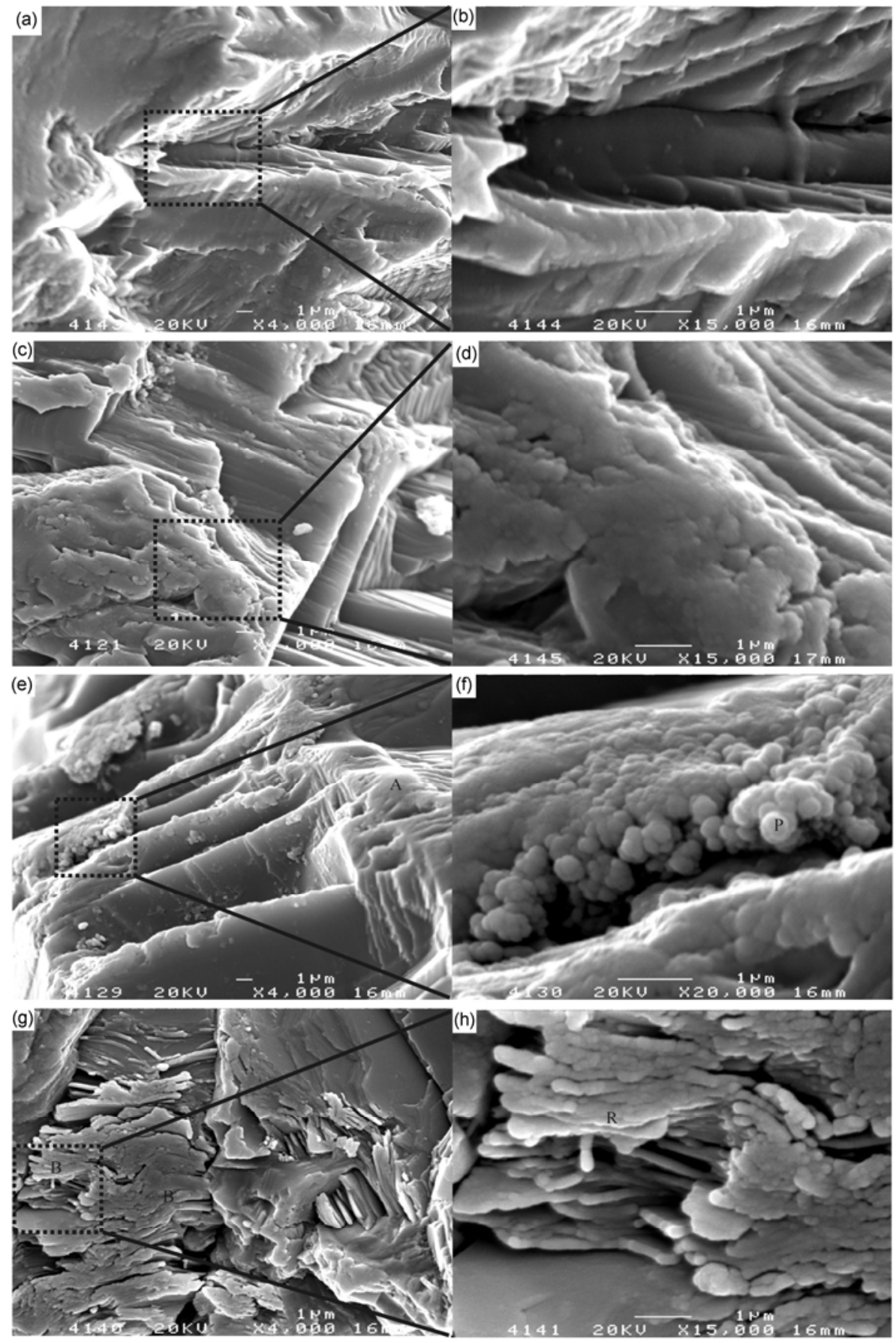

图 3 溶解前后钻长石表面形貌特征

(a) 溶解前; (b) (a)的局部放大; (c) 无菌 10 天; (d) (c)的局部放大(表面出现少许溶蚀现象); (e) 接触 10 天(表面高能位出现较强烈的溶解); (f) (e)的局部放大(接触实验中产生纳米级次生沉淀); ( g ) 透析 10 天(表面高能位出现较强烈溶解); ( h $)(\mathrm{g})$ 的局部放大(原来的薄片/ 板状物质溶解后残余大量相互串连的纳米级颗粒). A, B 分别为接触和透析实验中溶解较强烈的位置; P, 沉淀; R, 溶解残余

实验中则不存在类似沉淀. 由于这些次生沉淀物的 粒径(100 200 nm)大于透析袋孔径(约 $25 \AA$ 丹而小于菌 体尺寸 (约 $0.5 \mu \mathrm{m} \times 2 \mu \mathrm{m})^{[32]}$, 推断其可能为以细胞碎
片、细菌的表面官能团及其高分子量代谢物等为成核 物质的沉淀物. 这表明在细菌-矿物-溶液体系中，细菌 及其代谢物对矿物的溶解和次生沉淀的形成均具有促 


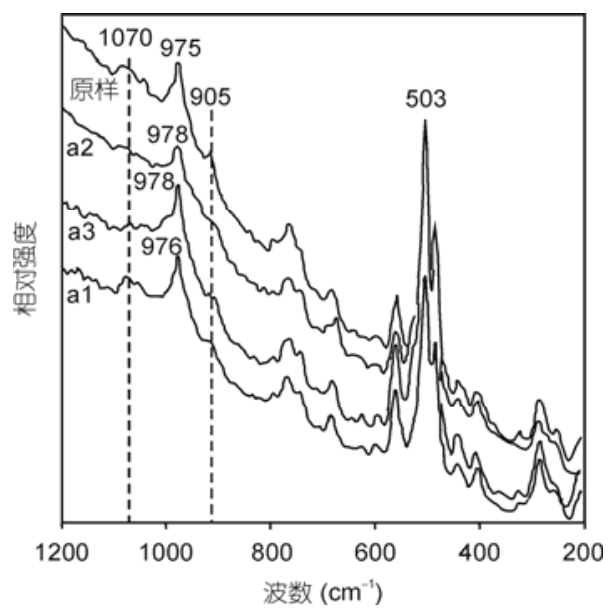

图 4 溶解前后钙长石表面的拉曼光谱

进作用.

实验结束时, 三组实验溶液中 $\mathrm{Ca}, \mathrm{Al}, \mathrm{Si}$ 浓度的 摩尔比分别为: 7.5/0.5/1, 0.9/0.6/1 和 1.7/0.5/1, 而原 样中三种元素的原子数比为 $0.4 / 0.8 / 1^{[22]}$, 由此可见, 如果不考虑沉淀消耗, 则三组实验中 $\mathrm{Ca}$ 的溶出相对 于 $\mathrm{Si}$ 都是较快的, 由此可能导致了长石表面的贫钙, 这与前人的研究是一致的 ${ }^{[33]}$. 对 $\mathrm{Al}$, 单从浓度比值 上看其溶出能力不如 $\mathrm{Si}$, 但是前人研究认为长石在 酸、有机配体及微生物的作用下 $\mathrm{Al}$ 通常优先于 $\mathrm{Si}$ 溶 出 $^{[33 \sim 36]}$, 这与元素浓度分析得出的结论相反. 因此, 需要寻找更多的证据, 对此现象做进一步的解释, 而 本研究试图通过拉曼光谱结果进行解析.

根据 Lasaga 和 Gibbs ${ }^{[37]}$ 的量子力学计算, 长石的 拉曼光谱在 $0 \sim 300 \mathrm{~cm}^{-1}$ 波段内的峰属于晶体格架的 联合振动, $300 \sim 700 \mathrm{~cm}^{-1}$ 间的峰属于 $\mathrm{Si}-\mathrm{O}-\mathrm{Si}$ 键的弯曲 振动, 700 1200 $\mathrm{cm}^{-1}$ 间的峰属于 $\mathrm{T}-\mathrm{O}(\mathrm{T}$ 为 $\mathrm{Si}$ 或 $\mathrm{Al}$ ) 的伸缩振动. Velde 和 Boyer ${ }^{[38]}$ 进一步将 700 1200 $\mathrm{cm}^{-1}$ 范围的峰归为硅(铝)氧四面体中桥氧或非桥氧 的振动. 通常情况下, 拉曼峰的强度与物质的含量有 关, 而峰位的变化与晶格的内应力有关. 因此, 根据 905 和 $1070 \mathrm{~cm}^{-1}$ 处峰的属性及其相对强度的减弱可 知微生物作用下长石表面的硅(铝)氧四面体结构发 生了较强的破坏. 有研究表明 ${ }^{[39]}$, 架状铝硅酸盐在高 频波段的峰位会随 $\mathrm{Al} / \mathrm{Si}$ 比的减小向高波数偏移. 相 对于原样, 细菌作用后的长石在 $975 \mathrm{~cm}^{-1}$ 处的峰发生 了蓝移, 加之峰强的变化, 表明长石表面发生了 $\mathrm{Al}$ 相对于 $\mathrm{Si}$ 的亏损. 这种微生物作用下 $\mathrm{Al}$ 相对于 $\mathrm{Si}$ 亏损的溶解特征, 与前人的实验是相符的. 由此可见,
通过 $\mathrm{Al}, \mathrm{Si}$ 浓度比和拉曼光谱结果得出不同结论的原 因, 是在考察 $\mathrm{Al}$ 和 $\mathrm{Si}$ 比时, 忽略了沉淀的影响. 当沉 淀的量较大, 同时 $\mathrm{Al}$ 比 $\mathrm{Si}$ 具有更高的沉淀效率时, 溶液中 $\mathrm{Al} / \mathrm{Si}$ 的值便不能真实反映长石中元素溶出的 优先性.

在长石中, $\mathrm{Al}$ 通常是以铝氧四面体的形式存在 的, 与 $\mathrm{Si}$ 共同构成了长石的架状结构. 由于在长石中 $\mathrm{Al}$ 只以 Al-O-Si 键结构存在 ${ }^{[40]}$, 因此细菌代谢产物对 长石格架的溶解, 主要表现在对 Al-O-Si 键的破坏作 用上. 事实上, $975 \mathrm{~cm}^{-1}$ 处的峰与 Al-O-Si 的反对称伸 缩振动有关 ${ }^{[31,41]}$, 而 $1070 \mathrm{~cm}^{-1}$ 与 $\mathrm{Si}-\mathrm{O}-\mathrm{Si}$ 的反对称伸 缩振动有关, 细菌溶解后, “前者峰位发生偏移, 后者 无偏移” 的拉曼光谱特征已表明细菌及其代谢物优先 破坏 Al-O-Si 桥氧键, 随后才破坏 Si-O-Si 桥氧键.

综合前述讨论可知, 细菌 P. polymyxa 可以加速 䥻长石的溶解和次生沉淀的生成. 溶解为非等化学 的, $\mathrm{Ca}$ 和 $\mathrm{Al}$ 优先于 $\mathrm{Si}$ 溶出, 细菌及其代谢物首先破 坏钙长石晶体格架中的 Al-O-Si 键, 溶解后长石表面 形成了相对于 $\mathrm{Si}$ 贫 $\mathrm{Ca}$ 和 $\mathrm{Al}$ 的淋滤层.

\section{2 细菌-长石相互作用机制}

细菌 P. polymyxa 的代谢产生多种有机酸(如甲酸、 乙酸、乳酸、柠檬酸等)和多聚糖 ${ }^{[24,25]}$, 这些物质通常能 以质子交换和配体络合方式促进长石的溶解 $[5,11 \sim 14,34,42]$. 已有研究表明 P. polymyxa 能有效溶解铝土矿中的含 $\mathrm{Ca}$ 和 $\mathrm{Fe}$ 杂质矿物相, 如方解石、针铁矿、赤铁矿等 ${ }^{[23]}$, 细菌的接触和不接触机制均起作用. 但是, 目前关于 该类细菌与铝硅酸盐矿物的相互作用机制研究还不 多见.

在无机条件下，当 $\mathrm{pH}<5.5$ 时，长石的溶解速率 通常随酸性增强而增大 ${ }^{[12,43]}$; 而在含有机酸体系中, 当 $\mathrm{pH}$ 在 3.5 8.5 之间时, 有机物中的基团(如羒基)会 通过配体络合作用显著加速其溶解 ${ }^{[12,44]}$. 在本研究 中, 有菌实验的 $\mathrm{pH}$ 值保持在 4.0 5.0 之间(图 1), 而 细菌及其代谢产物包含丰富的多种有机基团, 因此 质子交换和配体络合两种溶解形式都应存在. 在本 研究中, 如果不考虑沉淀消耗, 则各元素的浓度变化 速率可以被认为即是其溶出速率. 表 1 的结果表明, 无论是接触还是透析实验, $\mathrm{Ca}$ 的溶出速率在 $18 \mathrm{~h}$ 后 均逐渐降低, $\mathrm{Al}$ 和 $\mathrm{Si}$ 则保持恒定. 而在这一阶段, $\mathrm{pH}$ 的变化特征为先降低后升高. 由此可见, $\mathrm{Ca}$ 的溶出速 率与 $\mathrm{pH}$ 变化具有相关性, 该过程是主要通过质子交 
换作用进行的. 这一结果与前人的研究相同 ${ }^{[12,45 \sim 47]}$, 即在酸性溶液中, 长石中平衡电荷的碱金属和碱土 金属元素通常会首先通过质子交换作用而溶出. 由 于 $\mathrm{pH}$ 的变化并未影响 $\mathrm{Al}$ 和 $\mathrm{Si}$ 的溶出速率(无菌条件 下的吸附 $\mathrm{H}^{+}$也没有改变两种元素的溶出速率), 因此 两种元素的溶出速率主要与配体络合作用有关. 事 实上, 对比接触和透析实验结果(图 2), 也可进一步 证明该推断的正确性. 接触实验中细菌及多聚糖与 矿物接触机会多, 络合作用更普遍, 因此 $\mathrm{Al}$ 和 $\mathrm{Si}$ 的 溶出能力要强于透析实验中. 这种络合作用既包括 小分子量代谢物的络合 (透析实验中也有 $\mathrm{Al}$ 和 $\mathrm{Si}$ 溶 出量的上升), 也包括细菌的粘附导致的络合 (接触实 验中 $\mathrm{Al}$ 和 $\mathrm{Si}$ 溶出量更大). 而由于激光拉曼光谱已表 明粘附会导致长石中的 Al-O-Si 键首先遭到破坏, 说 明配体会优先络合 $\mathrm{Al}$ 和通过桥氧与 $\mathrm{Al}$ 连接的 $\mathrm{Si}$.

鉴于 $\mathrm{H}^{+}$对 $\mathrm{Al}$ 和 $\mathrm{Si}$ 溶出的参与度不大, 因此, 有 菌实验后期 $\mathrm{pH}$ 的上升, 便只可能与 $\mathrm{Ca}$ 的溶出消耗了 酸有关, 即 $\mathrm{Ca}$ 主要通过质子交换作用溶出. 由于细 菌在生长稳定期和衰亡期产酸能力较差, 因此当耗 酸能力超过产酸能力时, 溶液 $\mathrm{pH}$ 上升, $\mathrm{pH}$ 上升的结 果, 是使得质子交换能力变弱, $\mathrm{Ca}$ 溶出速率降低. 此 外, 需要注意的是, 样品处理时残留的“吸附性 $\mathrm{H}^{+}$” 在 有菌实验中对 $\mathrm{Ca}$ 的溶出也是有贡献的, 因此, 如果 没有这种 $\mathrm{H}^{+}$离子的存在, 可以显见的是, 实验结束 后溶液 $\mathrm{pH}$ 会上升更多, 而 $\mathrm{Ca}$ 的溶出量会更低.

由图 2 的曲线形状及表 1 的拟合方程可见, 对 $\mathrm{Ca}, \mathrm{Al}$ 和 $\mathrm{Si}$ 三种元素, 接触实验和透析实验中其溶出 模式均相同, 细菌及其代谢物的粘附没有改变三种 元素的溶出机制, $\mathrm{Ca}$ 仍然以质子交换为主, $\mathrm{Al}$ 和 $\mathrm{Si}$ 仍以配体络合为主, 只是粘附作用促进或抑制了这 两种机制.

\section{3 细菌粘附作用对长石溶解动力学的影响}

对 $\mathrm{Ca}$ 的溶出, 在不考虑沉淀情况下的两组微生 物实验中, 由 $\mathrm{Ca}$ 浓度随时间的变化均具对数特征可 见, $\mathrm{Ca}$ 的溶出速率随反应时间增长而降低(表 1), 此 时 $\mathrm{Ca}$ 的溶出反应不是零级反应, 溶出受反应物浓度 的影响. 由于 $\mathrm{Ca}$ 主要通过质子交换方式溶出, 因此 对透析实验, 制约 $\mathrm{Ca}$ 溶出的因素包括两方面: $\mathrm{pH}$ 值 和长石表面可溶性 $\mathrm{Ca}$ 的浓度. 在实验后期, 由于 $\mathrm{pH}$ 有所升高, 导致了溶液对 $\mathrm{Ca}$ 溶出能力的降低, 此时 $\mathrm{Ca}$ 的溶出受表面反应控制; 又由于淋滤层的形成,
导致 $\mathrm{Ca}$ 在长石表面的含量下降, 这种含量下将也必 将降低 $\mathrm{Ca}$ 的溶出速率, 这种淋滤层导致溶解速率降 低的动力学模式通常被称作“淋滤层扩散模式, [40,48]. 因此, 对透析实验, Ca 溶出除了受表面反应控制外, 还受淋滤层扩散的影响, 与无机酸条件下长石中碱金 属及碱土金属溶出速率的控制机制相同 ${ }^{[40]}$. 对接触实 验, $\mathrm{Ca}$ 的溶出模式与透析实验相同, 只是其浓度增长 速率不如透析实验, 推测细菌的粘附抑制了 $\mathrm{Ca}$ 的溶 出. 一方面, 细菌及其代谢物在长石表面的粘附, 不 利于 $\mathrm{Ca}$ 向溶液中扩散, 导致 $\mathrm{Ca}$ 在细菌-长石界面的微 环境中积聚, 抑制了钙长石溶解的持续进行; 另一方 面，这种微环境还会阻止溶液中的 $\mathrm{H}^{+}$离子到达长石表 面, 相当于长石的有效反应面积减少 ${ }^{[4,5,9,49]}$. 因此, 在 细菌-矿物接触的条件下, $\mathrm{Ca}$ 的溶出仍主要受表面反 应和淋滤层扩散控制, 而粘附的细菌会通过抑制表 面反应和元素扩散来影响 $\mathrm{Ca}$ 的溶出动力学.

对 $\mathrm{Al}$ 和 $\mathrm{Si}$ 的溶出动力学, 无论是在接触还是透 析实验中, 当不考虑沉淀消耗时, $\mathrm{Al}$ 和 $\mathrm{Si}$ 具有相同的 浓度增长模式, 溶出速率均不随时间而变化, 推测二 者的释出反应为两种可能的动力学模式: (1) 零级反 应, 此时长石表面 $\mathrm{Al}$ 和 $\mathrm{Si}$ 的浓度及络合物的浓度均对 溶出速率无影响, $\mathrm{Al}$ 和 $\mathrm{Si}$ 的溶出只受表面反应控制; (2) 非零级反应, 但此时表面 $\mathrm{Al}$ 和 $\mathrm{Si}$ 浓度变化对速率 的影响被与之络合的物质的浓度变化等因素的影响所 抵消, 致使 $\mathrm{Al}$ 和 $\mathrm{Si}$ 的溶出在表观上仍表现为只受表面 反应控制. 对比接触和透析实验结果知道, 细菌的粘 附加速了该动力学过程, 但未改变其溶出的控制模式. 尽管业已表明有菌实验中长石表面会出现 $\mathrm{Al}$ 相对于 $\mathrm{Si}$ 的亏损, 但这种亏损也未改变 $\mathrm{Al}$ 的溶出动力学.

从前述实验结果已知, 无论是接触还是透析实 验, 微生物作用均能导致长石表面形成富 $\mathrm{Si}$ 的淋滤 层, 因此, 对本研究使用的长石, 与非生物酸性条件 下相同, 其在微生物作用下溶解的速控步, 仍然是 $\mathrm{Si}$ 的溶出 ${ }^{[37,50]}$.

\section{4 结论}

本文通过使用透析方法开展对比实验研究, 探 讨了微生物及其高分子量代谢物在长石表面的粘附 对钙长石溶解的影响, 获得了一些关于长石微生物 溶解的粘附效应的新认识: (1) 在微生物粘附的微环 境中长石中各元素的溶出机制不会发生改变, 碱金 
属和碱土金属的溶出仍以质子交换为主, $\mathrm{Al}$ 和 $\mathrm{Si}$ 的 溶出仍以配体络合为主, 配体络合作用优先破坏架 状结构中的 Al-O-Si 键; (2) 细菌的粘附会抑制质子 交换反应，抑制碱金属和碱土金属的溶出，但能促进 配体络合反应，加速 $\mathrm{Al}$ 和 $\mathrm{Si}$ 的溶出; (3) 细菌及其代
谢产物作用下，长石中碱(土)金属的溶出主要受表面 反应和淋滤层扩散控制, 粘附的细菌会通过抑制表 面反应和元素扩散来影响其溶出动力学; $\mathrm{Al}$ 和 $\mathrm{Si}$ 的 溶出则只受表面反应控制, 细菌的粘附可以加速该 动力学过程.

致谢南京大学地球科学与工程学院周新民教授和徐夕生教授提供了本研究所需的地质样品, 拉曼光谱工作由该 学院王天刚完成, 南京农业大学周立祥教授和审稿专家对本文的修改提出了非常宝贵的意见, 在此表示衰心 感谢.

\section{参考文献}

1 Hazen T C, Jimenez L, de Victoria G L. Comparison of bacteria from deep subsurface sediment and adjacent groundwater. Microb Ecol, 1991, 22: 293-304

2 Holm P E, Nielsen P H, Albrechtsen H J, et al. Importance of unattached bacteria and bacteria attached to sediment in determining potentials for the degradation of xenobiotic organic contaminants in an aerobic aquifer. Appl Environ Microbiol, 1992, 58: 3020-3026

3 Ehrlich H L. How microbes influence mineral growth and dissolution. Chem Geol, 1996, 132: 5-9

4 Ullman W J, Kirchman D L, Welch S A, et al. Laboratory evidence for microbially mediated silicate mineral dissolution in nature. Chem Geol, 1996, 132: 11-17

5 Welch S A, Ullman W J. The effect of microbial glucose metabolism bytownite feldspar dissolution rates between $5^{\circ} \mathrm{C}$ and $35^{\circ} \mathrm{C}$. Geochim Cosmochim Acta, 1999, 63: 3247-3259

6 Wilson M J. Weathering of the primary rock-forming minerals: Processes, products and rates. Clay Mineral, 2004, 39: 233-266

7 陆建军, 陆现彩, 王睿勇, 等. 多金属矿山环境中矿物的微生物分解及环境效应研究进展. 高校地质学报, 2007, 13: 621-629

8 Lian B, Wang B, Pan M, et al. Microbial release of potassium from K-bearing minerals by thermophilic fungus Aspergillus fumigatus. Geochim Cosmochim Acta, 2008, 72: 87-98

9 Hiebert F K, Bennett P C. Microbial control of silicate weathering in organic-rich ground water. Science, 1992, 258: 278-281

10 Barker W W, Welch S A, Chu S, et al. Experimental observations of the effects of bacteria on aluminosilicate weathering. Am Mineral, 1998, 83: 1551-1563

11 Bennett P C, Melcer M E, Siegel D I, et al. The dissolution of quartz in dilute aqueous solutions of organic acids at $25^{\circ} \mathrm{C}$. Geochim Cosmochim Acta, 1988, 52: 1521-1530

12 Amrhein C, Suarez D L. The use of a surface complexation model to describe the kinetics of ligand-promoted dissolution of anorthite. Geochim Cosmochim Acta, 1988, 52: 2785-2793

13 Wieland E, Wehrli B, Stumm W. The coordination chemistry of weathering: III. A generalization on the dissolution rates of minerals. Geochim Cosmochim Acta, 1999, 52: 1969-1981

14 Sterflinger K. Fungi as geologic agents. Geomicrobiol J, 2000, 17: 97-124

15 Casey W H, Bunker B. Leaching of mineral and glass surfaces during dissolution. Rev Mineral, 1990, 23: 397-425

16 Muir I J, Bancroft G M, Shotyk W, et al. A SIMS and XPS study of dissolving plagioclase. Geochim Cosmochim Acta, 1990, 54: $2247-2256$

17 Hellmann R. The albite-water system: Part I . The kinetics of dissolution as a function of $\mathrm{pH}$ at 100,200 , and $300^{\circ} \mathrm{C}$. Geochim Cosmochim Acta, 1994, 58: 595-611

18 Hellmann R. The albite-water system: Part II. The time evolution of the stoichiometry of dissolution as a function of pH at 100, 200, and $300^{\circ}$ C. Geochim Cosmochim Acta, 1995, 59: 1669-1697

19 Gout R, Oelkers E H, Schott J, et al. The surface chemistry and structure of acid-leached albite: New insights on the dissolution mechanism of the alkali feldspars. Geochim Cosmochim Acta, 1997, 61: 3013-3018

20 肖奕, 王汝成, 陆现彩, 等. 低温碱性溶液中微纹长石溶解性质研究. 矿物学报, 2003, 23: 333-340

21 Kohler S J, Bosbach D, Oelkers E H. Do clay mineral dissolution rates reach steady state? Geochim Cosmochim Acta, 2005, 69: 1997-2006

22 周新民, 徐夕生, 董传万, 等. 中国东南活动大陆边缘的矿物标志: 钻长石质斜长石. 科学通报, 1994, 39: 1011-1014

23 Anand P, Modak J M, Natarajan K A. Biobeneficiation of bauxite using Bacillus polymyxa: Calcium and iron removal. Int J Mineral Process, 
1996, 48: 51-60

24 Mankad T, Nauman E B. Effect of oxygen on steady state product distribution in Bacillus polymyxa fermentations. Biotechnol Bioeng, 1992, 40: 413-426

25 Santhiya D, Subramanian S, Natarajan K A. Surface chemical studies on sphalerite and galena using extracellular polysaccharides isolated from Bacillus polymyxa. J Coll Interf Sci, 2002, 256: 237-248

26 Bromfield S M. Reduction of ferric compounds by soil bacteria. J Gen Microbiol, 1954, 11: 1-6

27 Wightman P G, Fein J B. The effect of bacterial cell wall adsorption on mineral solubilities. Chem Geol, 2004, 212: 247-254

28 周跃飞, 王汝成, 陆现彩, 等. 微生物-矿物接触模式影响矿物溶解机制的实验研究. 高校地质学报, 2007, 13: 657-661

29 Welch S A, Barker W W, Banfield J F. Microbial extracellular polysaccharides and plagioclase dissolution. Geochim Cosmochim Acta, 1999, 63: 1405-1419

30 Hutchens E, Valsami-Jones E, McEldowney S, et al. The role of heterotrophic bacteria in feldspar dissolution—An experimental approach. Mineral Mag, 2003, 67: 1157-1170

31 Le Parc R, Champagnon B, Dianoux J, et al. Anorthite and $\mathrm{CaAl}_{2} \mathrm{Si}_{2} \mathrm{O}_{8}$ glass: Low frequency Raman spectroscopy and neutron scattering. J Non-crystal Solid, 1992, 323: 155-161

32 周跃飞, 王汝成, 陆现彩. 玄武岩微生物分解过程中的矿物表面效应. 岩石矿物学杂志, 2008, 27: 59-66

33 Oelkers E H, Schott J. Experimental study of anorthite dissolution and the relative mechanism of feldspar hydrolysis. Geochim Cosmochim Acta, 1995, 59: 5039-5053

34 黄思静, 杨俊杰, 张文正, 等. 不同温度条件下乙酸对长石溶蚀过程的实验研究. 沉积学报, 1995, 13: 7-17

35 Frogner P, Broman C, Lindblom S. Weathering detected by Raman spectroscopy using Al-ordering in albite. Chem Geol, 1998, 151: 161-168

36 Smith J V. Atmospheric weathering and silica-coated feldspar: Analogy with zeolite molecular sieves, granite weathering, soil formation, ornamental slabs, and ceramics. Proc Nat Acad Sci USA, 1998, 95: 3366-3369

37 Lasaga A C, Gibbs G V. Quantum mechanical potential surfaces and calculations on minerals and molecular clusters. Phys Chem Mineral, 1988, 16: 29-41

38 Velde B, Boyer H J. Raman microprobe spectra of naturally shocked microcline feldspars. J Geophys Res, 1985, 90: 3675-3682

39 Neuville D R, Cormier L, Massiot D. Al environment in tectosilicate and peraluminous glasses: A ${ }^{27} \mathrm{Al}$ MQ-MAS NMR, Raman, and XANES investigation. Geochim Cosmochim Acta, 2004, 68: 5071-5079

40 Ribbe P H. Chemistry, structure and nomenclature of feldspars. Rev Mineral, 1983, 2: 1-19

41 Sharma S K, Simons B, Yoder H S. Raman study of anorthite, calcium Tschermak's pyroxene, and gehlenite in crystalline and glassy states. Am Mineral, 1983, 68: 1113-1125

42 向廷生, 蔡春芳, 付华娥. 不同温度、羧酸溶液中长石溶解模拟实验. 沉积学报, 2004, 22: 597-602

43 Drever J I, Stillings L L. The role of organic acids in mineral weathering. Coll Surf A, 1997, 120: 167-181

44 罗孝俊, 杨卫东. 有机酸对长石溶解度影响的热力学研究. 矿物学报, 2001, 21: 183-188

45 Muir I J, Nesbitt H W. Controls on differential leaching of calcium and aluminium from labradorite in dilute electrolyte solutions. Geochim Cosmochim Acta, 1992, 56: 3979-3985

46 Muir I J, Nesbitt H W. Reactions of aqueous cations at the labradorite-water interface: Coupled effect of surface processes and diffusion. Geochim Cosmochim Acta, 1997, 61: 265-274

47 Oxburgh R, Drever J, Sun Y T. Mechanism of plagioclase dissolution in acid solution at $25^{\circ} \mathrm{C}$. Geochim Cosmochim Acta, 1994, 58: 661-669

48 Casey W H, Ludwig C. Silicate mineral dissolution as a ligand-exchange reaction. Rev Mineral, 1995, 31: 87-117

49 Welch S A, Vandevivere P. Effect of microbial and other naturally occurring polymers on mineral dissolution. Geomicrobiol J, 1994, 12: $227-238$

50 Oelkers E H. General kinetic description of multioxide silicate mineral and glass dissolution. Geochim Cosmochim Acta, 2001, 65: 3703-3719 